\title{
The Forgotten Brick: Case Report of a Lisfranc Injury with "Pericuneiform" Dislocation
}

\author{
Helen Bumann* and Martin Wonerow
}

Department of Orthopaedics and Traumatology, Regional Hospital Surselva, Ilanz, Switzerland

*Corresponding author: Dr. med. Helen Bumann, Department of Orthopaedics and Traumatology, Regional Hospital Surselva, Spitalstrasse 6, 7130 Ilanz, Switzerland, Tel: +41-926-73-72

\begin{abstract}
Introduction: The Lisfranc joint complex forms the transverse arch of the foot. The Lisfranc joint complex is fundamental for both stability and flexibility of the foot, whereas the intermediate cuneiform anchors as its keystone. Due to the complexity of the joint, Lisfranc injuries show many different patterns. Up to now, there is limited evidence regarding treatment recommendations. Furthermore, as shown in our case, some injury patterns do not fit into existing classification.
\end{abstract}

Case: A 42-year-old patient presented himself after a motorcycle accident. A CT-scan showed intra-articular comminuted fractures of the cuboid bone, intermediate cuneiform and lateral cuneiform. The intermediate cuneiform was dorsally dislocated. We decided to perform a combined fracture treatment with primary arthrodesis of the second and third tarsometatarsal joints and plate osteosynthesis of the cuboid bone. Three months postoperatively, the patient was painfree and able to work $100 \%$ in a physically demanding profession.

Discussion: Anatomical reduction of the Lisfranc joint line is crucial in displaced Lisfranc injuries. Our particular case could not been fitted into existing guidelines. Considering biomechanical factors, we guaranteed the stability of the "roman arch" by performing an arthrodesis of the second and third tarsometatarsal joints while preserving midfoot flexibility through osteosynthesis of the cuboid bone.

Conclusion: In addition to already existing recommendations, biomechanical understanding of the Lisfranc joint complex should be taken into consideration when choosing treatment. Preservation of the stability of the second tarsometatarsal joint and-contrarily-flexibility of the fourth and fifth tarsometatarsal joints should be treated as priorities.

Level of clinical evidence: level 4 clinical evidence.

\section{Introduction}

Injuries to the Lisfranc joint complex accountfor approximately $0.2-0.9 \%$ of all fractures [1]. Firstdescribed by Malgaine in 1856, the anatomically corresponding structures of the Lisfranc injuries received their name from Jacques Lisfranc, who, as a field surgeon under Napoleon in 1815, used tarso-metatarsal amputation as a treatment for forefoot gangrene [2]. The Lisfranc joint includes two joint lines: The distal joint line consists of the bases of the metatarsal bones, while the proximal joint line is formed by the ossa cuneiformia and the os cuboideum. The joints between the distal and proximal joint linesand - functionally of utmost significance - their various ligaments belong to the Lisfranc joint complex as well [2].

Strong plantar and interosseous ligaments stabilise the entire complex, while the dorsal ligamentous apparatus is only weakly developed. The interosseous ligaments connect both the bases of the metatarsalia to each other and the bases of the metatarsalia to the corresponding tarsal bones [2]. A well-known exception is the Lisfranc ligament, which runs in a Y-shaped form from the medial cuneiform to the bases of the first and second metatarsals; an intermetatarsal ligament is missing between these two metatarsal bases [2,3].

Together, the structures belonging to the Lisfranc joint complex form the so-called "roman arch", the transverse arch of the foot. The base of the second metatarsal, together with its strong plantar ligamentous structures, anchors itself as the keystone between the medial and lateral cuneiform bones and is thus fundamental for the stability of the entire arch 
[4]. This is also reflected by its rigidity: In the first and second tarsometatarsal joints, which anatomically form a tight amphiarthrosis, studies showed only wobbling movements. In contrast, movement amplitudes of 5-10 were found in the first tarsometatarsal joint and $10-20^{\circ}$ in the fourth and fifth tars metatarsal joints $[2,4]$. These facts considered, it can be concluded that the first, second and third tars metatarsal joints are primarily responsible for the stability of the joint complex. The comparatively greater mobility of the fourth and fifth tarsometatarsal joint, on the other hand, give the foot flexibility, such as the ability to adapt to uneven ground [4].

Both low-energy and high-energy trauma can lead to Lisfranc joint injury, which can be caused by both direct and indirect mechanisms $[5,6]$. Correct diagnosis at the time of accident plays a crucial role, as untreated injuries can lead to chronic instability and early osteoarthritis in up to $50 \%$, particularly in cases of primary instability [1].

Due to the relative rarity of the injury as well as the complexity of the joint complex and therefore highly variable injury patterns, it is difficult to develop treatment recommendations or guidelines.

It can be agreed upon the importance of restoring the congruency of the joint line [7]. However, there is controversy about whether to treat the injuries with ORIF or primary arthrodesis. Henning, et al. showed no difference in functional outcomes between primary ORIF vs. primary arthrodesis in a prospective randomised trial with 40 participants [6]. In contrast, Qiao, et al. 2017 favored primary arthrodesis over osteosynthesis in a retrospective study with 25 participants [8].

\section{Classification}

Up to now, the classification according to Hardcastle, which is essentially based on the classification of Quenu and Küss from 1909, has been the most widely accepted. Quenu distinguished Lisfranc joint injuries by three types, homolateral, divergent and isolated [9]. Hard castle expanded the classification describing subtypes, each specifying the direction of the fracture luxation, e.g. medial or lateral [10]. However, neither the dorsoplantar level nor the proximal parts of the Lisfranc series are taken into account in the injury pattern in both the Quenu and Hardcastle classifications. Therefore, if there are dislocation injuries within the proximal Lisfranc line, these cannot be classified according to existing classifications. The same applies to partial dorsoplantar dislocations of the distal row $[9,10]$ (Figure 1).

\section{Specific controversy in our case}

In our case, in addition to the fractures, there was an isolated dorsal dislocation of the intermediate cuneiform in relation to all adjacent articular partners due to injury to the dorsal and plantar ligaments.
A

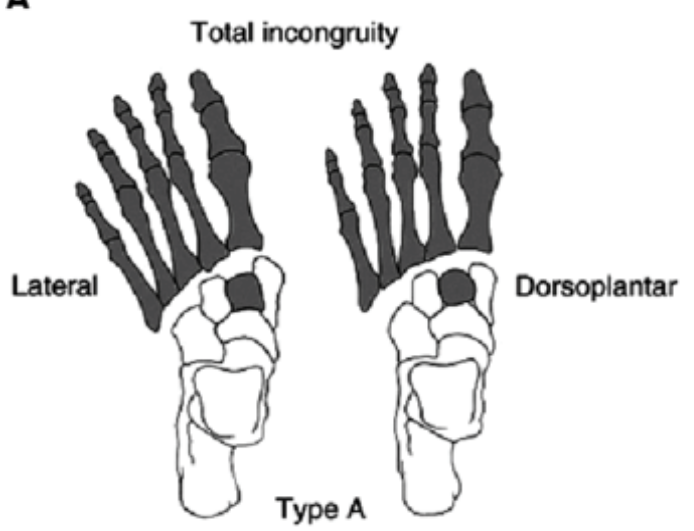

C
B

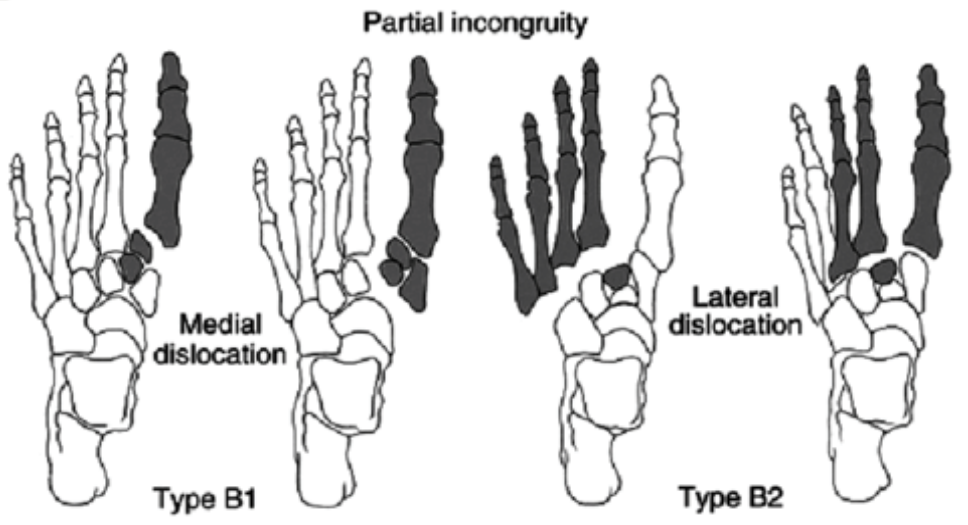

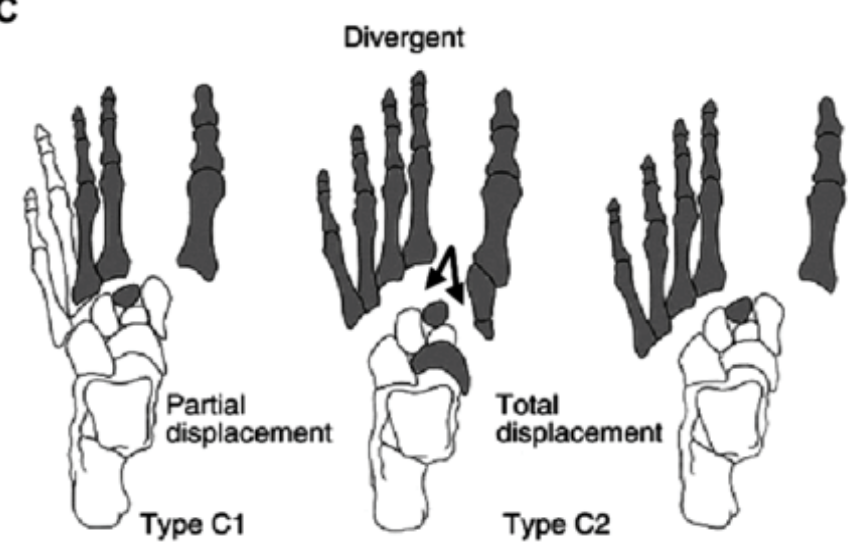

Figure 1: Hardcastle \& Myerson classification of Lisfranc-injuries. Graphic from [9]. 


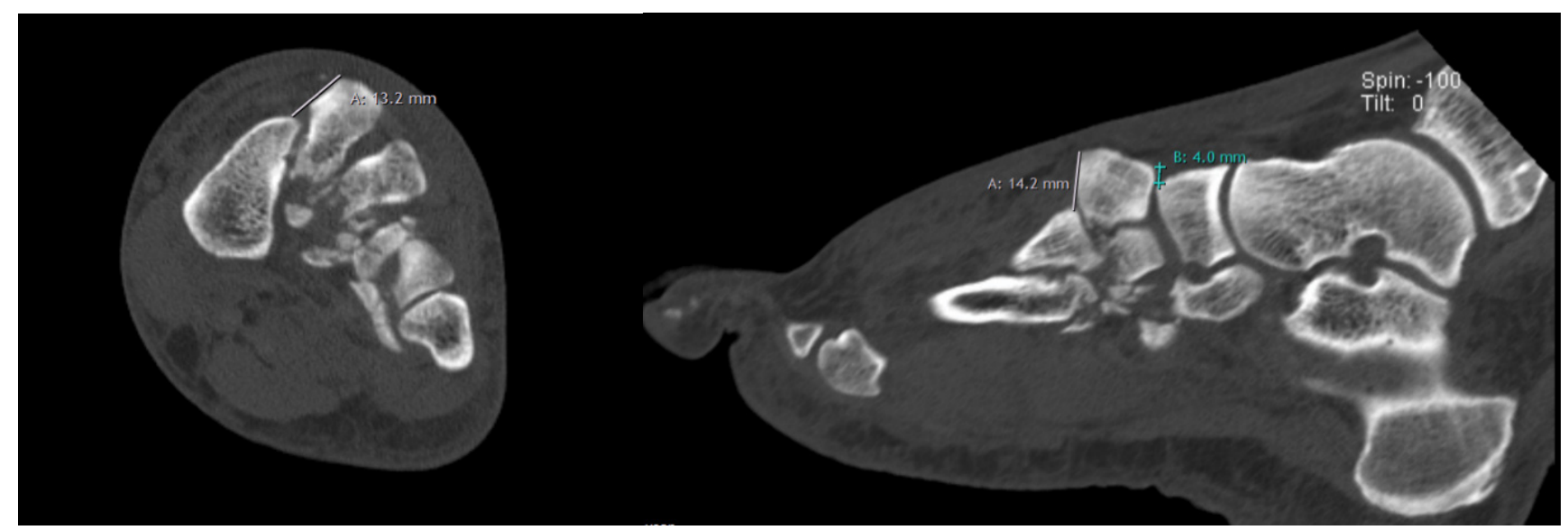

Figure 2: Pre-operative axialand sagittal CT-cuts show a dorsal dislocation of the intermediate cuneiform of $14 \mathrm{~mm}$.

Following the definition of perilunate dislocation in the wrist, in which there is also a tearing of the palmar and dorsal ligaments with subsequent luxation of the surrounding joint partners, one can speak of a pericuneiform dislocation [11].

In the literature, only a few cases of dorsal dislocation of the intermediate cuneiform have yet been described in the form of case reports. Within this group of sparsely described injuries, the accompanying ligamentous and osseous injuries and therefore their specific treatments are quite varying, including the usage of distractor, K-wires, plates and screws and as well closed and open reduction [12-15]. Thus, a consensus or treatment recommendation cannot be derived [16].

\section{Case}

\section{History/clinic/diagnostics}

A 42-year-old patient presented himself with massive pain, swelling and weight-bearing intolerance of the left foot after a motorbike accident. Having slipped on his motorbike in a curve, the motorbike had fallen directly on his left foot. X-rays taken in the ER already showed a Lisfranc dislocation fracture. A CT-scan was subsequently performed to assess the fracture, whereby multifragmentary, intra-articular comminuted fractures involving all articular surfaces of the cuboid bone, intermediate cuneiform and lateral cuneiform could be revealed. The intermediate cuneiform was dorsally dislocated in relation to all adjacent articular partners. In addition, there were multi-fragmentary intra-articular base fractures of the third and fourth metatarsals and a slightly dislocated head fracture of the third metatarsal. Due to soft tissue swelling, the lower leg was primarily immobilised in a cast until the operation could be performed (Figure 2).

\section{Fracture treatment}

Owing to the complexity of the injury in the area of the second and third tarsometatarsal joints as well as the dislocation of the intermediate cuneiform, we decided to perform arthrodesis of the second and third tarsometatarsal joints and between the intermediate cuneiform and navicular bone. The approach was chosen dorsally between the second and third metatarsal.

In order to preserve the mobility of the fourth and fifth tarsometatarsal joints and thus enable a safe and stable gait even on uneven terrain, it was decided to do an open reduction and internal fixation on those two joints.

The intermediate cuneiform was reduced manually without any problems. After removal of the cartilage of the joint partners, the arthrodesis of the second metatarsal, the intermediate cuneiforme and the navicular bone was done with a head compression screw and cancellous bone graft (extraction site: medial malleolus). To secure rotational stability, a locking cloverleaf plate was fitted and fixed from the navicular bone over the intermediate cuneiform to the base of the second metatarsal.

The third tarsometatarsal joint was prepared for arthrodesis with the same method and afterwards bridged using a locking L-plate.

The open reduction and internal fixation of the cuboid bone was done via a second approach. After anatomical reduction of the comminuted fracture of the cuboid bone with fragments going into the fourth and fifth tarsometatarsal articular surfaces, the fracture was fixed with a slightly pre-bent locking cuboid plate (Figure 3).

\section{Outcome}

For follow-up treatment, the patient was initially immobilised in a lower leg cast (VACOped). After the swelling of the soft tissues had subsided, the immobilisation was switched to a lower leg plaster cast. Clinical and radiological controls were carried out after 6 and 10 weeks and after 6 months. After 6 weeks, with increasing blurring in the area of the arthrodesis gap, it was possible to change from the lower leg plaster cast to 

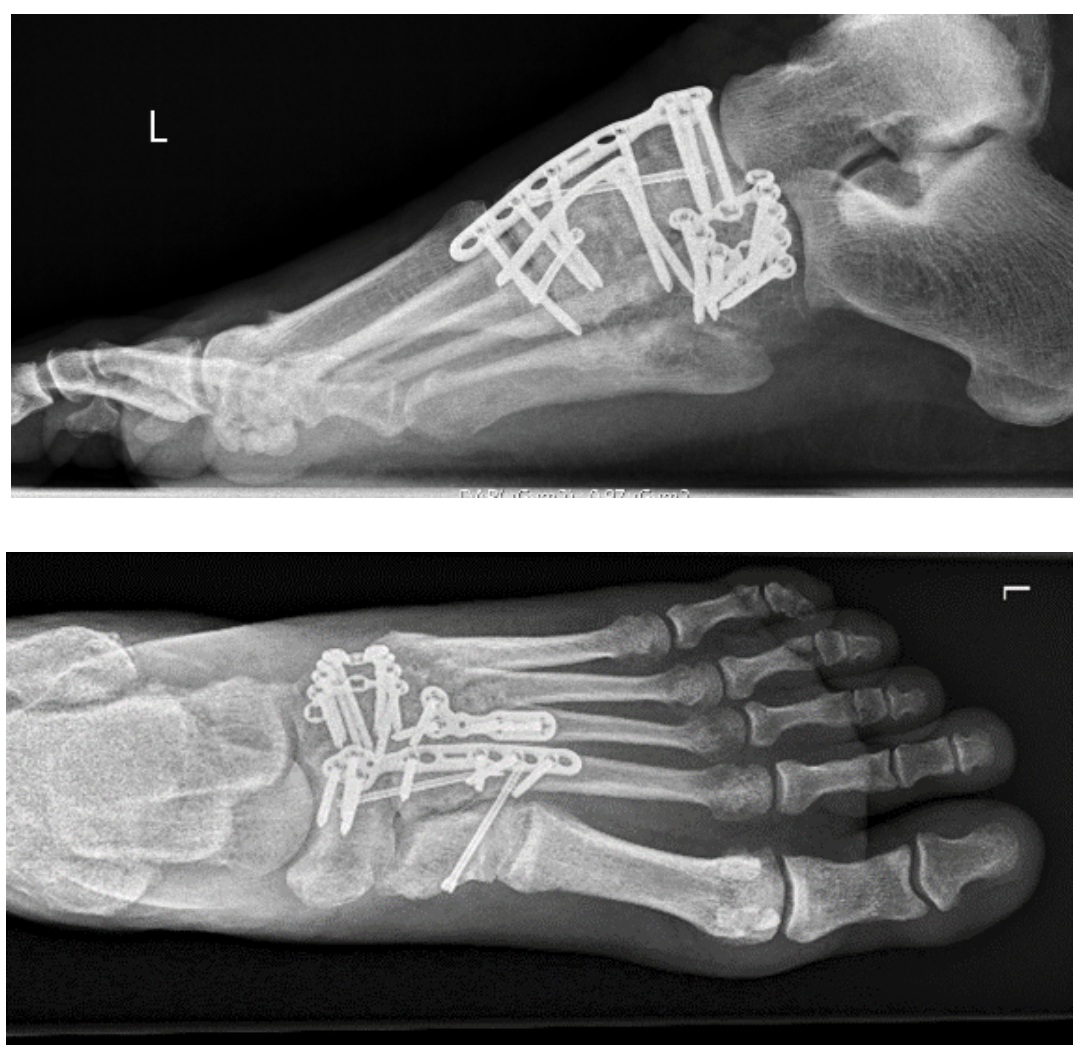

Figure 3: Post-operative X-rays ap/lat.

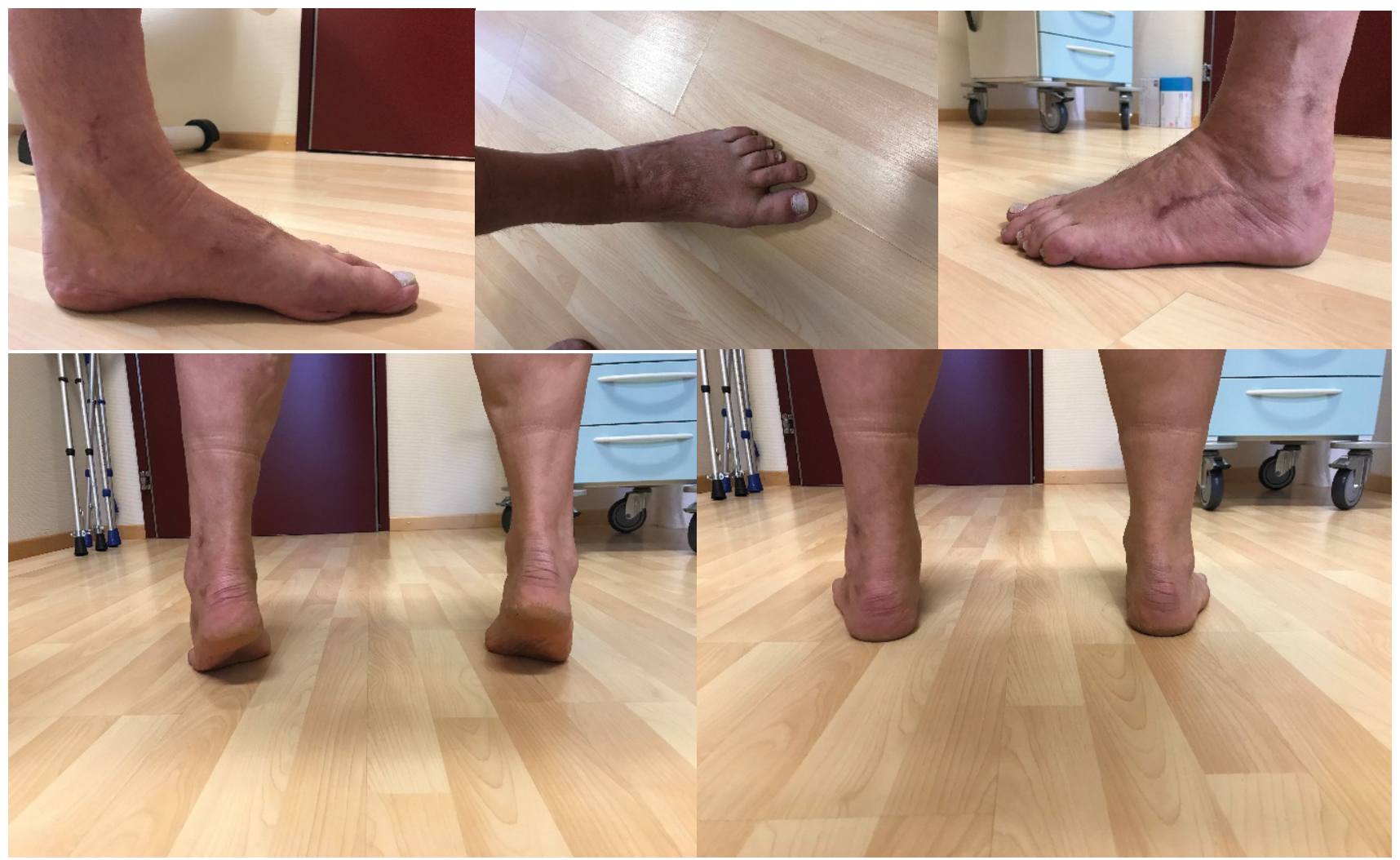

Figure 4: Clinical outcome 5 months postoperatively.

VACOped again, and after 10 weeks full weight-bearing was started.

The patient was able to work $100 \%$ in a profession with physical activity from 14 weeks postoperatively. The patient was even able to do physical work on ladders without any discomfort3 months postoperatively. For the final examination after 5 months, a CT-scan showed a fusion from the second metatarsal to the navicular bone. Apart from a slight tendency to swelling, which had already regressed, the patient was symptom-free. 


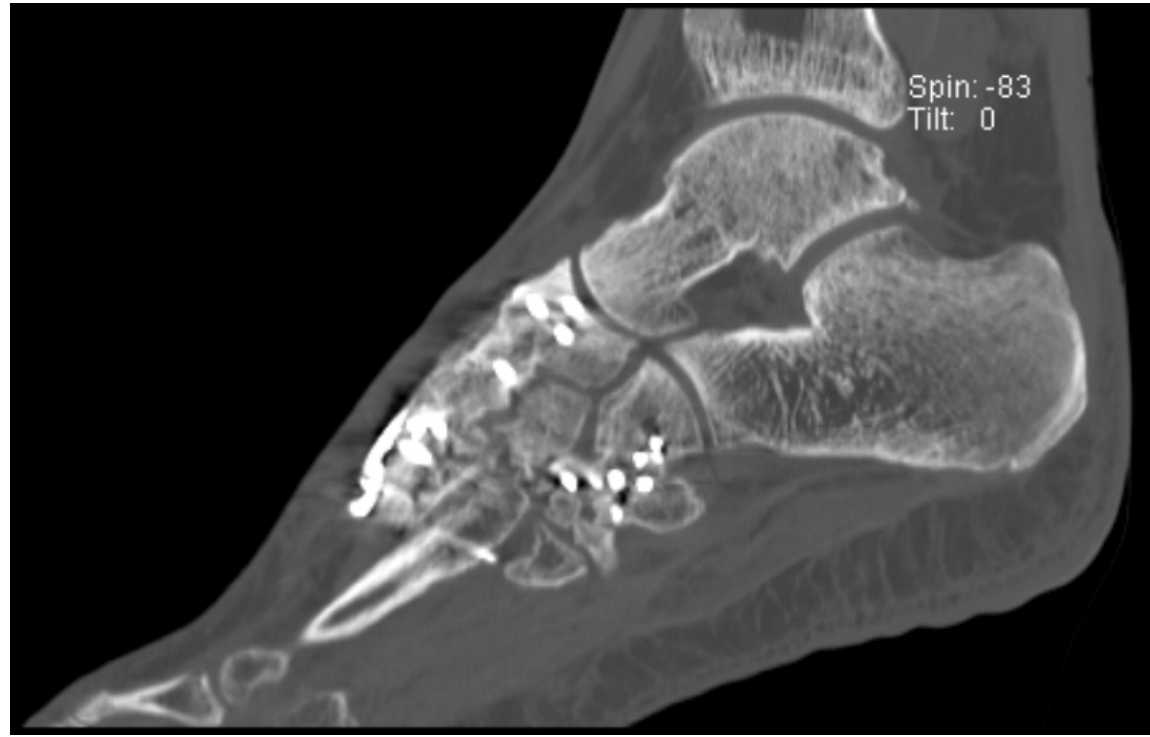

Figure 5: CT-scan of the foot showing fusion from the second metatarsal to the navicular bone.

The upper ankle joint dorsiflexion/plantar flexion was symmetrical to the opposite side at $20 / 0 / 40^{\circ}$. Forefoot abduction and adduction against resistance was $\mathrm{M} 5 / 5$, the same applies to dorsiflexion. The Lisfranc and Chopart joint lineswere painfree on palpation and twisting. The patient did not take any pain-killersfrom the third postoperative week onwards (Figure 4 and Figure 5).

\section{Discussion}

An anatomical position of the Lisfranc joint line even with weight-bearing is crucial in Lisfranc injuries and a requirement of conservative therapy. Whenever, and quite often in the case of less obvious, primarily ligamentous injuries, an unstable injury is diagnosed, surgical treatment is clearly indicated. However, data on the outcome between primary arthrodesis versus open reduction and internal fixation are controversial in the literature [17].

In a review from 2019, Moracia-Ochagavía, et al. elaborated a management approach recommending unstable osseous injuries for ORIF and ligamentous injuries for primary arthrodesis [5].

The recommendations, like the vast majority of other authors do, are based on the established classification of Hardcastle \& Myerson. As described above, our particular case could not been fitted into those guidelines due to a lack of classification of proximal dislocation injuries [10]. To make a decision, we therefore sought other parameters to apply, whereby two considerations proved to be determining:

On the one hand, through the dislocation of the intermediate cuneiform, the dorsal ligamentous apparatus was ruptured, which, as described above, is weakly developed and therefore cannot be repaired [2]. On the other hand, the second tarsometatarsal joint forms an amphiarthrosis. Consequently, the preservation of its stability as a keystone in the "Roman arch" is much more important than the relatively insignificant loss of the poor mobility in this joint [4]. In a synopsis of these factors, the primary arthodesis with preservation of stability was considered a priority and thus the preferred treatment of the medial column. To minimise the risk of pseudarthrosis in the area of arthrodesis, we used a combination of compression screw and locking plate for fixation $[18,19]$.

\section{Conclusion}

Injuries of the Lisfranc joint complex are very variable due to their complexity and therefore sometimes difficult to classify; recommendations for surgical treatment cannot directly be applied to all injuries. An extension of the existing classification to include dislocation injuries within the proximal Lisfranc joint line and partial dorsoplantar dislocations of the distal joint line would be desirable in order to achieve better decision-making recommendations. The selection criteria between primary arthrodesis vs. ORIF should mainly be based on biomechanical considerations, whereby, in addition to the crucial anatomical reduction, stability in the second tarsometatarsal joint and, in contrast, the functionality of the fourth and fifth tarsometatarsal joints should be considered as priorities.

\section{Conflicts of Interest}

The authors declare no conflict of interest.

\section{Informed Consent}

Informed Consent was obtained from the patient.

\section{References}

1. De Orio M, Erickson M, Usuelli FG, Easley M (2009) Lisfranc Injuries in Sport. Foot Ankle Clin 14: 169-186.

2. Wirth CJ (2002) Orthopädie Und Orthopädische Chirurgie Fuss. Thieme. 
3. Zwipp H, Rammelt S (2014) Anatomische Wiederherstellung des chronisch instabilen Lisfranc-Ligaments. Unfallchirurg 117: 791-797.

4. Coetzee JC (2008) Making Sense of Lisfranc Injuries. Foot Ankle Clin 13: 695-704.

5. Moracia Ochagavía I, Rodríguez-Merchán EC (2019) Lisfranc fracture-dislocations: Current management. EFORT Open Rev 4: 430-444.

6. Henning JA, Jones CB, Sietsema DL, Bohay DR, Anderson JG (2009) Open reduction internal fixation versus primary arthrodesis for lisfranc injuries: A prospective randomized study. Foot Ankle Int 30: 913-922.

7. Lau S, Bozin M, Thillainadesan T (2017) Lisfranc fracture dislocation: A review of a commonly missed injury of the midfoot. Emerg Med J 34: 52-56.

8. Qiao Y-S, Li J-K, Shen H, Bao H-Y, Yan Liu, et al. (2017) Comparison of Arthrodesis and Non-fusion to Treat Lisfranc Injuries. Orthop Surg 9: 62-68.

9. Myerson M (1989) The diagnosis and treatment of injuries to the Lisfranc joint complex. Orthop Clin North Am 20: 655664.

10. Mahmoud S, Hamad F, Riaz M, Ahmed G, Al Ateeq M, et al. (2015) Reliability of the Lisfranc injury radiological classification (Myerson-modified Hardcastle classification system). Int Orthop 39: 2215-2218.

11. Martini AK (2003) Orthopädie Und Orthopädische Chirurgie Ellenbogen, Unteram, Hand. Thieme.
12. Doshi D, Prashanth Prabhu AB (2008) Dorsal dislocation of the intermediate cuneiform with fracture of the Lisfranc joint: A case report. J Foot Ankle Surg 47: 60-62.

13. Asuma MP, Mansfield TD, Turner EK, Robins J (2019) Closed distal dislocation of the intermediate cuneiform in a complex lisfranc fracture-dislocation: A case report. JBJS 9: e0332.

14. Kalraiya AJ, Vanhegan I, Cheung A, Rudge B (2014) A rare lisfranc-type injury involving dorsal dislocation of the intermediate cuneiform. BMJ Case Rep.

15. Singh AP, Singh AP, Garg V, Garcha JS (2016) Dislocation Intermediate Cuneiform with Fracture Medial Cuneiform. J Orthop case reports 6: $32-34$.

16. Akan B, Yildirim T (2013) Dorsal Dislocation of the Intermediate Cuneiform with a Medial Cuneiform Fracture: A Case Report and Review of the Literature. Case Rep Orthop 2013: 238950.

17. Stavlas P, Roberts CS, Xypnitos FN, Giannoudis PV (2010) The role of reduction and internal fixation of Lisfranc fracture-dislocations: A systematic review of the literature. Int Orthop 34: 1083-1091.

18. DeVries JG, Granata JD, Hyer CF (2011) Fixation of first tarsometatarsal arthrodesis: A retrospective comparative cohort of two techniques. Foot Ankle Int 32: 158-162.

19. Plaaß C, Claaßen L, Ettinger S, Daniilidis K, StukenborgColsman C (2017) Lapidus-Arthrodese. Orthopäde 46: 424433. 